\title{
Acute Retropharyngeal Calcific Tendinitis or Calcium Hydroxyapatite Deposits in Longus Colli Muscle
}

\author{
Adrian Gallego-Goyanes ${ }^{\mathrm{a}, \mathrm{b}}$, Laura Anta-Martinez ${ }^{\mathrm{a}}$, \\ Jesus Pino-Minguez ${ }^{\mathrm{a}}$
}

\begin{abstract}
Acute retropharyngeal calcific tendinitis, also called calcium hydroxylapatite deposition disease, is a little known entity that causes pain and neck stiffness associated with odynophagia. Radiographically, it manifested with calcium deposits and an edematization of retropharyngeal soft tissues. Retropharyngeal impacting typically occurs to the long neck muscle tendon insertion or longus colli, although it is rarely described, in the literature. We present a clinical case treated in the Department of Traumatology and Orthopaedic Surgery of Hospital Clinico Universitario of Santiago de Compostela (La Coruna, Spain), discussing the clinical-radiological characteristics and treatment according to the little existing literature on the subject. After the analysis of the data obtained from the review of this case, we can conclude that the most important element for the diagnosis of this entity is the knowledge of its existence and recognition of its characteristics in plain X-ray and CT. It is self-limited and her treatment consists of anti-inflammatory agents, non-steroidal and neck brace immobilization.
\end{abstract}

Keywords: Calcium hydroxylapatite; Acute calcific; Extensor longus colli; Retropharyngeal abscess; Retropharyngeal tendinitis

\section{Introduction}

Acute retropharyngeal calcific tendinitis, also called calcium hydroxylapatite deposition disease, is a little known entity that causes pain and neck stiffness associated with odynophagia [1, 2]. Radiographically, it manifested with calcium deposits and a dropsy of the retropharyngeal soft tissues. The mechanism of its formation is not fully enlightened, attributing this more recently to genetic and metabolic factors, rather than traumatic factors as described initially [1,3-5].

Manuscript accepted for publication March 20, 2014

aDepartment of Traumatology and Orthopaedic Surgery, Hospital Clinico Universitario of Santiago de Compostela, La Coruna, Spain

bCorresponding Author: Adrian Gallego Goyanes, Complejo Hospitalario Universitario de Santiago de Compostela, Servicio de COT, La Coruna, Spain. Email: Adrian.gallego.goyanes@gmail.com

doi: https://doi.org/10.14740/jmc1650w
The most frequent locations of acute calcifying tendinitis are the extremities and within the shoulder. Retropharyngeal impacting typically occurs to the long neck muscle tendon insertion or longus colli, although it is rarely described in the literature $[4,6,7]$.

The long neck, the main muscle flexor of the cervical spine, consists of three parts: inferior oblique, upper oblique and vertical, this muscle is located ahead of first cervical vertebrae $[6,8,9]$. It typically occurs at the top level of C1-C2. The deposit of hydroxyapatite in the sinewy muscle insertion may result in symptomatic tendinitis which is often diagnosed accidentally, since it has an atypical clinic neck pain associated with swallowing pain [4, 7, 9-11].

We present a case of acute retropharyngeal tendinitis with calcium deposit and describe the radiological characteristics and treatment according to the little existing literature on the subject.

\section{Case Report}

The patient was a 50-year-old woman without a personal history of interest except for appendectomy, cholecystectomy and frequent neck pain without underlying pathology. She had no usual treatment at home, smoked four cigarettes/day and did not drink alcohol.

The patient was admitted to the Emergency Department of the Hospital Universitario of Santiago de Compostela with 3 days of right neck pain without prior injury and different characteristics from which they suffer on a regular basis. The day after the presentation, she went to her physician for primary care that consisted in immobilizing her neck with a soft cervical collar, and some drugs (diazepam, AINEs), with poor clinical improvement. Then she decided to go to the emergency room because of the pain and suddenly odynophagia and dysphagia for solids and liquids. She also had difficulty for the mouth opening, feeling of cervical inflammation, no changes in voice and no fever at his home. Concomitant upper tract infection was not presented in this process.

Upon arrival to the emergency room, she had low-grade fever $\left(37.4{ }^{\circ} \mathrm{C}\right)$, strong neck pain with important limitation of cervical mobility, odynophagia and dysphagia. There was limitation of cervical mobility especially in the extension, with right para-tracheal tenderness that increased with swallowing 

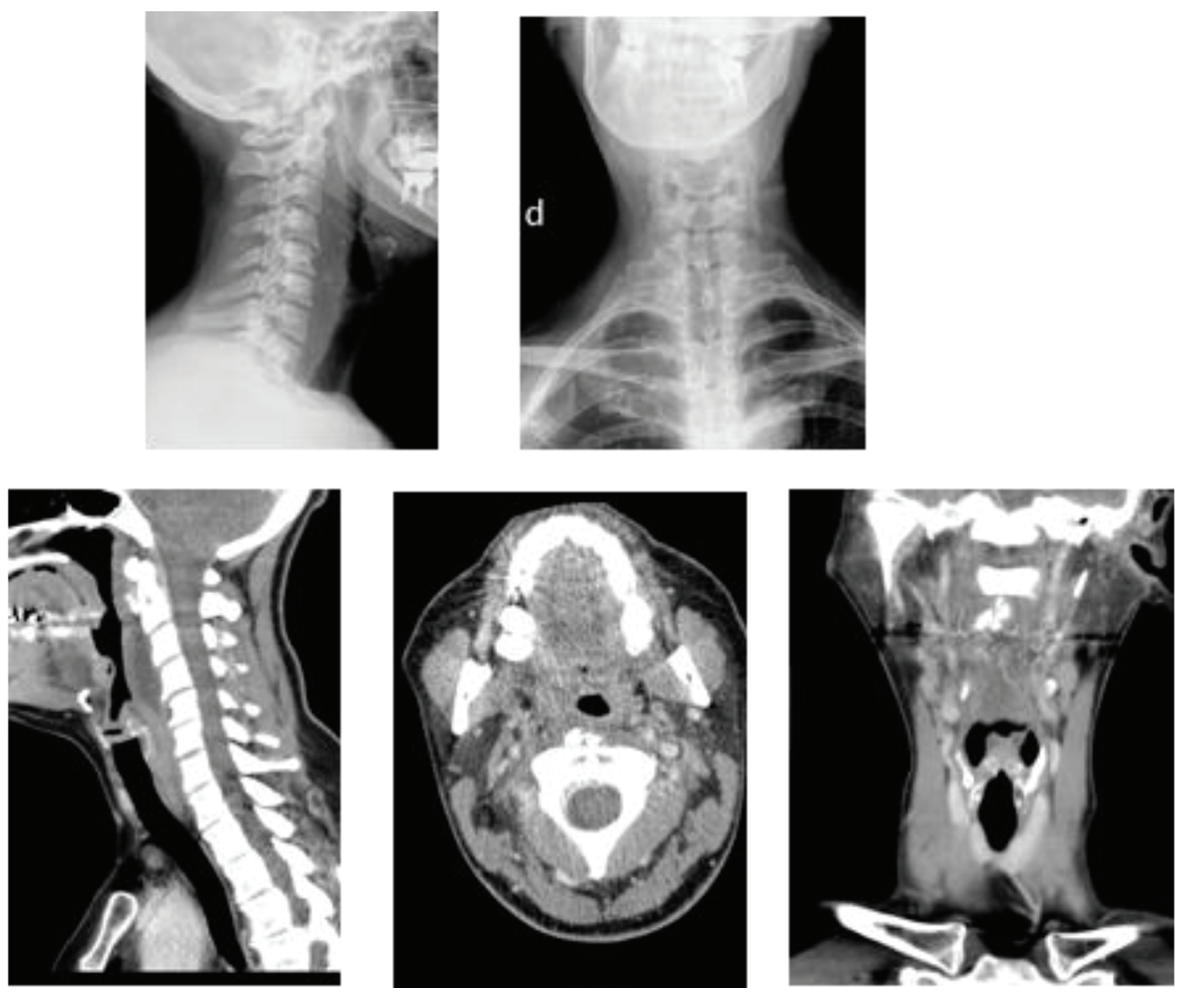

Figure 1. Two images of cervical plain on top. The first side showing rectification of physiological lordosis next to the prevertebral calcifications at level C1-C2 and edematization of the prevertebral space. Pictures of CT on the bottom showing moderate increase of prevertebral soft tissues at the height of C2, C3 and C4 and a tenuous calcification of irregular morphology, approximately $15 \times 14.5 \times 9.5 \mathrm{~mm}$ in intimate relation with the proximal portion of the longus colli muscle belly.

and cervical paravertebral muscles contraction. There were no tender lymph nodes and oropharyngeal examination performed by otorhinolaryngologists of guard was rigorously normal. Moderate leukocytosis and increased ESR were detected. Blood cultures and urine cultures were performed, as well as $\mathrm{X}$-rays of posteroanterior and lateral cervical spine at the suspicion from retropharyngeal infectious process.

After a simple cervical X-ray, a loss of cervical physiological lordosis and widening of the prevertebral soft tissues associated with an amorphous focal calcification prior to the first and second cervical vertebrae were observed (Fig. 1a, b). In computerized tomography (CT) scan of neck, we noted moderate increase of prevertebral soft tissue at the height of $\mathrm{C} 2, \mathrm{C} 3$ and $\mathrm{C} 4$ without evidence of the presence of organized abscess collections showing in location immediately preceding the basis of process odontoid process and lower anterior atlas arc. Dim calcification of irregular morphology of this was approximately $15 \times 14.5 \times 9.5 \mathrm{~mm}$ in intimate relation with the proximal portion of the longus colli muscle belly (Fig. 1c, d and e), providing us the diagnosis of prevertebral calcific tendinitis.

Given that the patient had very disabling symptoms, hospital admission was decided for corticosteroids, anti-inflammatory non-steroidal, cervical immobilization with soft collar and monitoring of the process. Two days after his hospital admission, a complete clinical improvement without X-rays of the calcification disappearance was observed. Guideline to the high anti-inflammatory drugs was cited 2 weeks into the inquiry. Figure 2 shows clinically asymptomatic radiograph with reduction of calcification in the lateral cervical.

\section{Discussion}

Acute retropharyngeal calcific tendinitis is a clinical syndrome first described by Hartley [1] in 1964, which was demonstrated by Ring et al [2] in 1994 as secondary to deposition of calcium hydroxyapatite in the long neck muscle. The longus colli muscle consists of three parts: superior oblique, inferior oblique and vertical. Classically, the calcification affects the superior oblique portion of muscle longus colli at level C1-C2. Acute prevertebral calcific tendinitis may go unnoticed and be misdiagnosed due to its rare occurrence [1-4].

The acute retropharyngeal calcific tendinitis is a form of disease by deposition of calcium hydroxylapatite which specifically involves the extensor longus colli and longus tendon of the big toe. This is a rare location for a disease that typically involves large joints such as the hips or shoulders. The etiology of calcium hydroxylapatite crystals deposit is not clear. It is believed that in the time that calcium crystals have been deposited within the extensor longus colli muscle, it cause a pain due to the rupture of these crystals which can cause a painful inflammatory response to foreign bodies in the long neck muscle which would lead to the formation of reactive fluid in the 

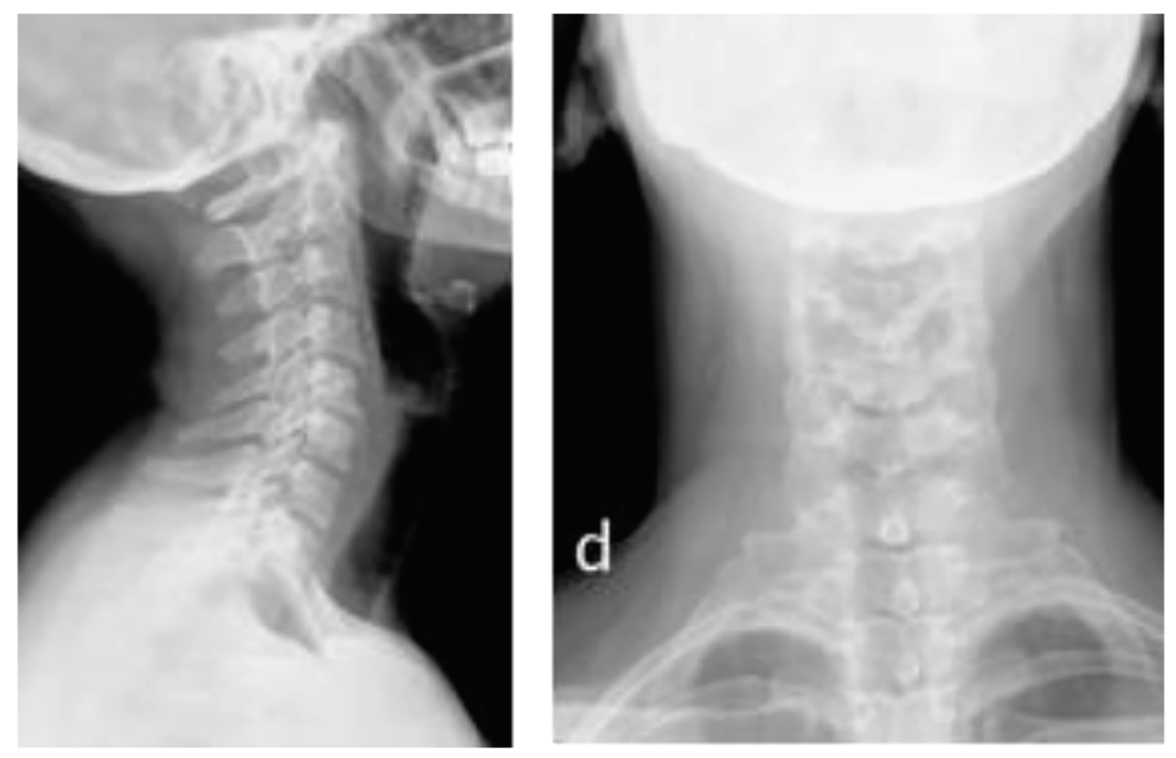

Figure 2. Two simple cervical X-ray images are shown at 2 weeks of evolution. The first side shows again physiological lordosis and a disappearance of the prevertebral calcifications at level C1-C2 and decrease of edema of the prevertebral space.

prevertebral space surrounding the muscle $[2,5-7]$.

Condition affects adults within a reported age range of $21-81$ years, with greater distribution between 30 and 60 years. The symptoms are secondary to inflammation of long extensor muscle of the neck and include an acute to subacute onset of pain in the neck, dysphagia, odynophagia and fever of low grade [5-9]. Dysphagia is due to the proximity of the retropharyngeal space to the adjacent pharyngeal constrictor muscles. It produces no shortness of breath. The patient may have a low-grade fever, as well as be quietly altered leucocyte and globular sedimentation speed. All this could mistakenly target a retropharyngeal infection. However, in the latter, the patient usually presented greater breach of general state with marked fever and alteration of the differential and acute phase reactants. A thorough anamnesis and clinical exploration is therefore fundamental $[9,10]$.

The typical pain occurs mainly in the anterior cervical region with contracture of the muscles paravertebral rear component and no irradiation. The pain increases with mobility, being very limited, and increases with the expansion and improvement with the flexion of the neck.

Oropharyngeal in these patients is completely normal inspection and odynophagia pertaining to patients is typically lateralized [10-13].

The main radiographic findings include swollen prevertebral soft tissue, and previous amorphous calcification to rent C1-C2. The amorphous and diffuse thickening of the prevertebral soft tissue typically extends from $\mathrm{C} 1$ to $\mathrm{C} 4$, while you can extend below to C6.

Although this condition is rare, its true incidence is probably higher, since it is frequently not diagnosed by ignorance of many clinicians, misrepresentation and confusion with other entities. The progressive accessibility of emergency services CT will probably allow document new cases [4-6].

The thickening of the soft tissue represents either spill dis- crete or diffuse edema, which can be differentiated in the CT or MRI. The lack of improvement that surrounds the spill may be useful in reactive differentiation of a spill from an abscess. CT can help identify calcium deposits, which often may seem weak on plain X-ray. Magnetic resonance imaging (MRI) is not so useful as CT for detecting calcifications due to its less sensitivity. Besides, MRI is not always available in Emergency Department. However, it would show an increase of signal in T2 to edema in the retropharyngeal space and occasionally could demonstrate bone edema in the vert.

It is a self-limiting disease which can resolve spontaneously after $1-2$ weeks. If it is not, it is self-limited and resolves within several weeks of the onset of symptoms, while it is quite painful and debilitating for the patient during that time. Some forms of conservative treatment, such as a short course of antiinflammatory steroid-inflammatory and the avoidance of aggravating the neck movements, can help relieve the symptoms. In severe cases, intravenous corticosteroids provide a speedy clinic recovery $[3,6,8]$.

Knowing the strange thing of this entity and its pathognomonic radiological findings can avoid incorrect medical therapy and invasive attempts as surgical drains. On the other hand, to be aware of this disease, we can save the patient from unnecessary diagnostic, anxiety and delays in discharge from undue hospital [1-3].

\section{Conclusion}

The most important element for the diagnosis of this entity is the knowledge of its existence and recognition of its characteristics in plain X-ray and CT. MRI, being of help, is less than CT scans in diagnostic capability due to their low sensitivity to detect calcium deposits.

No definition of calcifications in the soft parts on the sim- 
ple X-ray may lead to other diagnoses. So the implementation of CT is recommended if clinical suspicion is high and the Xrays show no calcification.

It is a self-limiting disease which can resolve spontaneously after 1 - 2 weeks. Knowing the strange thing of this entity and its typical radiological findings, they do that we can avoid incorrect medical therapy and invasive attempts as surgical drains, opting for a simple treatment with no steroidal anti-inflammatory drugs, non-steroidal and neck brace immobilization.

\section{References}

1. Hartley J. Acute Cervical Pain Associated with Retropharyngeal Calcium Deposit. A Case Report. J Bone Joint Surg Am. 1964;46:1753-1754.

2. Ring D, Vaccaro AR, Scuderi G, Pathria MN, Garfin SR. Acute calcific retropharyngeal tendinitis. Clinical presentation and pathological characterization. J Bone Joint Surg Am. 1994;76(11):1636-1642.

3. Razon RV, Nasir A, Wu GS, Soliman M, Trilling J. Retropharyngeal calcific tendonitis: report of two cases. J Am Board Fam Med. 2009;22(1):84-88.

4. Leep Hunderfund AN, Robertson CE, Bell ML, Busby DJ, Koehler TF, Ireland SP. Calcific retropharyngeal tendinitis: unusual cause of acute neck pain with nuchal rigidity. Neurology. 2008;71(10):778.

5. Omezzine SJ, Hafsa C, Lahmar I, Driss N, Hamza H. Cal- cific tendinitis of the longus colli: diagnosis by CT. Joint Bone Spine. 2008;75(1):90-91.

6. Sanghvi DA, Jankharia BG, Purandare NC, Sundaram M. Radiologic case study. Acute calcific retropharyngeal tendinitis. Orthopedics. 2006;29(7):561, 650-561.

7. Queinnec S, Petrover D, Guigui P, Ilharreborde B. Benign febrile cervicalgia due to calcific retropharyngeal tendinitis: case study. Orthop Traumatol Surg Res. 2011;97(3):341-344.

8. Shin DE, Ahn CS, Choi JP. The acute calcific prevertebral tendinitis: report of two cases. Asian Spine J. 2010;4(2):123-127.

9. Silva CF, Soffia PS, Pruzzo E. Acute prevertebral calcific tendinitis: a source of non-surgical acute cervical pain. Acta Radiol. 2014;55(1):91-94.

10. Gabra N, Belair M, Ayad T. Retropharyngeal calcific tendinitis mimicking a retropharyngeal phlegmon. Case Rep Otolaryngol. 2013;2013:912628.

11. Horowitz G, Ben-Ari O, Brenner A, Fliss DM, Wasserzug $\mathrm{O}$. Incidence of retropharyngeal calcific tendinitis (longus colli tendinitis) in the general population. Otolaryngol Head Neck Surg. 2013;148(6):955-958.

12. Terao K, Kusunoki T, Mori K, Murata K, Doi K. A case of calcific retropharyngeal tendinitis: the significance of an early diagnosis. Ear Nose Throat J. 2013;92(2):74-83.

13. Boikov AS, Griffith B, Stemer M, Jain R. Acute calcific longus colli tendinitis: an unusual location and presentation. Arch Otolaryngol Head Neck Surg. 2012;138(7):676679. 